\title{
SÍNODOS EUROPEOS DE MUJERES. ESPIRITUALIDAD FEMINISTA EN ACCIÓN
}

\author{
Ma PilAR De Miguel FERNÁNDEZ \\ Universidad de Deusto
}

Recibido: $17 / 7 / 2012$

Aceptado 08/10/2012

\section{Resumen}

El término sínodo procede del griego y define a una asamblea de gente con la misma trayectoria y propósito. Este término se usó desde muy pronto en la comunidad cristiana para identificar los encuentros y los concilios de diversa importancia. Hoy día, el término va unido a un modo muy diferente de tomar decisiones en las estructuras eclesiásticas. En el cristianismo primitivo, las mujeres estaban representadas en todos los órdenes de la comunidad y se vivía un discipulado de iguales. El Sínodo Europeo de mujeres, que es un movimiento crítico, usa el término sínodo para reclamar la herencia histórica y religiosa de las mujeres y un modelo de toma de decisiones.

Palabras clave: sínodo, herencia, transmisión, memoria, toma de decisiones, movimiento crítico, espiritualidad política.

\begin{abstract}
The term synod is derived from Greek legal terminology and refers to an assembly of people with the same goal and the same path. The term was used very early to characterize the assembly of Christian women and men in community gathering and councils in small or larger circles. Today, the term synod is linked to very different power and decision-making structures in the individual Christian churches. In the early church, women were represented in all positions of the community, and early Christian communities lived in discipleship of equals. The European Women's Synod, which is understood to be a movement of critical seeking, uses the term synod to reclaim our historical and religious heritage.
\end{abstract}

Key words: synod, heritage, legacy, decisions-making, critic movement, feminist spirituality 

«El Reino de los Cielos es semejante a la levadura que tomó una mujer y la metió en tres medidas de harina, hasta que fermentó todo» ${ }^{1}$.

\section{Introducción}

Cuando terminó el Primer Sínodo europeo de mujeres en Gmunden (Austria) el 28 de julio de 1986 vino a mi memoria con fuerza algo que había oído y no siempre compartía pero que se me impuso con fuerza: un gramo de realización vale por toneladas de abstracción. La experiencia de la que brotaba esta convicción no era en absoluto que despreciara la abstracción; era algo así como: se podía, lo hemos hecho... la realización, no temer, ponerse manos a la obra frente a abstracciones de «podré o no podré», «seremos capaces o no» «me gustaría pero...» Las realizaciones concretas marcas hitos necesarios. La acción transforma. Quería remarcar sobre todo en estos momentos la acción porque se atisban confusiones entre lo virtual y lo real.

También he elegido dos acontecimientos de la teología feminista europea y de los movimientos de espiritualidad que esta genera porque reivindico también la memoria, y el reconocimiento de sus logros. La cultura feminista ha sido creada y vivida por millones de mujeres de carne y hueso de otras generaciones y contemporáneas de toda condición y en toda circunstancia. Muchas no se conocieron nunca e incluso pensaron que lo suyo no era de lo mismo. El reconocimiento, iqué gran tema!

Como dirá la antropóloga mexicana, Marcela Lagarde ${ }^{2}$

Desconocemos sus recorridos de vida porque los recursos de la memoria no las registraron, pero las adivinamos porque sabemos que cada lucha, convocatoria o movimiento se sostiene en decenas de miles, sumergidas e invisibles, que viven hasta en sin palabras lo que otras significan. Pero todas son mujeres que al vivir han abierto brechas, cambiado normas y subvertido su

1. (Mt 13,33).

2. Marcela LAGARDE, «Aculturación feminista» en www.geocities.com/litertulia/acultur_ mlagarde.htm 20/04/01, 7 
mundo inmediato. Con sus acciones cotidianas o excepcionales, trastocan el mundo de la mayoría.

Cada día, la cultura feminista se enfrenta al asunto de la transmisión de todo esto con complejo de Penélope, aunque más que haciendo y deshaciendo, sería haciendo mientras se lo deshacen. Como decíamos, lo dominante es que se privilegie las acciones que legitiman el patriarcado olvidando las aportaciones a la vida social y cultural que realizan las mujeres. La formación escolar y universitaria está estructurada en torno a esta visión de la historia y del mundo que enseña esta concepción genérica claramente parcial y por tanto incorrecta, ente otras cosas. "Todas las instituciones de nuestra cultura nos dicen a través de las palabras, de las acciones y, peor aún, de los silencios, que nosotras somos insignificantes. Pero nuestra herencia es nuestro poder» dirá la artista Judy Chicago.

Cuando pienso en el feminismo, veo una venerable y sabia anciana; con siglos de existencia, expresado multi-culturalmente y formulado de modos bastante políglotas. ¿Qué sucede que permanentemente encuentro personas e instituciones que la miran como si fuera una joven adolescente y balbuceante que inicia su proceso vital y de aprendizaje a tientas? Más aún, lo que observo es una mirada condescendiente esperando que esa joven impetuosa madure. No obstante me causa cierta perplejidad, por no emplear otros términos, la inconfesada recepción que se hace de esa aportación sin nombrarla, usurpando, de hecho estas aportaciones, invisibilizando la procedencia y renombrándolo acorde a los intereses; de modo que así siempre somos recién llegadas y como por sorpresa.

Vemos también como las iglesias se defienden negando e invisibilizando la gran recepción imparable que va teniendo lugar de la teología y espiritualidad feministas. Es algo con carácter nominalista. Lo prohibido es el nombre. Se pretende recibir «reeducándolo» porque el problema, y coincido con Meyer-Wilmes ${ }^{3}$ al respecto, no es lo bien o mal fundados que estén las propuestas que se aportan, sino la distinta cosmovisión que plantea la teología feminista. Si se nombra, es marginal junto con otras aproximaciones marginales, como las teologías étnicas o contextuales, etc. y sobre todo se dice que se percibe como partidista y excluyente.

Yo creo que la mayor formación de las mujeres, el desarrollo de diferentes tipos de feminismo y movimientos de mujeres y muy especialmente la evolución teológica en el campo de la teología feminista sentaron las bases de

3. H. MeYER-Wilmes, Rebellion on the Borders. Feminist Theology between Theory and Praxis, Pharos, Kampen-The Netherlands, 1995, pp. 186-187. 
una nueva conciencia eclesial que se despliega por cada continente y país y se extiende a todo el mundo ${ }^{4}$. Están estableciendo objetivos teológicos y los están viviendo juntas en toda su pluralidad. Es evidente que todas las iglesias de todas las religiones se están beneficiando de este dinamismo y por tanto la sociedad entera.

Se ha ofrecido y se ofrece toda la experiencia de llevar un tiempo transitando esos caminos de futuro Tal vez comiencen a cansarse de proporcionar un servicio de alta calidad a cambio de una recompensa de baja calidad.

\section{Sínodos europeos de mujeres. Contexto y claves para su comprensión}

La Conferencia de Naciones Unidas de 1985, que clausuraba en Nairobi El Decenio de la Mujer, instaba en su documento final a las Iglesias a que «supriman las enseñanzas y prácticas discriminatorias para con las mujeres, como una respuesta cristiana a las estrategias para el futuro», aprobadas por dicha conferencia. Esta interpelación la recoge el Consejo Ecuménico de las Iglesias que decreta un nuevo Decenio (1988-1998) de Solidaridad de las Iglesias con las Mujeres, proponiendo los siguientes objetivos:

a. La plena participación de las mujeres en la vida de la Iglesia y de la comunidad.

b. El compromiso de las mujeres por la paz, la justicia y la salvaguarda de la creación. Se concretó muy especialmente en la violencia contra las mujeres en la sociedad y en la iglesia; la injusticia económica y su impacto sobre las mujeres de todo el mundo y los efectos del racismo y la xenofobia sobre las mujeres.

c. La participación de las mujeres en la teología y en la comunicación espiritual.

Ese mismo año (1985), Anna Karin Hammar, entonces en el Comité Central del Consejo Mundial de las Iglesias, expresa por primera vez la necesidad de un Sínodo de Mujeres. Debido a que este nombre tiene una definición propia en la mayoría de las iglesias, hubo algunas resistencias contra su conveniencia. Sin embargo, fue decisivo para la adopción final del término, el componente que da a entender que las mujeres se entienden a sí mismas como seres humanos con facultad de decisión.

4. Amplio más esto en mi aportación, «Las iglesias cristianas en Europa ante el siglo XXI», en El Cristianismo ante el siglo XXI, una mirada nueva. Evangelio y liberación, Madrid, 2000. Actas XIX Congreso de Teología. 
Hay un grupo inicial que comienza a elaborar la idea de Sínodo europeo entre Holanda y Alemania, donde al igual que en otros países del entorno, organizan sínodos nacionales, que se ampliará posteriormente dando lugar al Comité Preparatorio Internacional, compuesto por mujeres de Holanda, Alemania, Gran Bretaña, Austria, Lituania, Hungría, Italia, Francia, España, Polonia, Rumania, República Checa, Croacia, Suecia y Suiza... Así llega 1996, año en que tiene lugar el Primer Sínodo europeo en Gmunden (Austria).

\section{Antecedentes}

El Concilio Vaticano II sirvió como catalizador de toda inquietud alrededor de la anómala situación de las mujeres en la Iglesia. Despertó gran entusiasmo que luego se vio bastante frustrado en este sentido. El concilio no se ocupó expresamente de este asunto. No obstante, por primera vez, un grupo de mujeres es invitado, tarde, eso sí, ya había habido alguna sesión, en la que el cardenal Suenens advirtió que media humanidad estaba fuera del aula conciliar. Pablo VI incorporó a la tercera sesión del concilio, aceptando así la interpelación del cardenal, a 23 mujeres (10 religiosas y 13 seglares), la mayoría de éstas elegidas entre las dirigentes de las organizaciones internacionales católicas. Esta situación insólita hasta el momento está cargada de anécdotas indescriptibles que ha transmitido con gracia Pilar Belosillo, una española entre las 13 seglares elegidas.

Todo el momento posterior es complejo. Lo que ahora quiero destacar es que en esta circunstancia se gesta una primera red de mujeres, diríamos hoy, que posteriormente y gracias a las relaciones establecidas, dentro del espíritu conciliar, y convocando a otras incluso de otras tradiciones cristianas (laicas, diaconisas, teólogas), constituyen un núcleo de diálogo ecuménico: cerca de Roma, en 1965.

Tras mucho trabajo y mucha historia entre medio, reflexiones y resultados tremendamente interesantes, este grupo será el antecesor del Foro Ecuménico de mujeres cristianas de Europa. El Foro se constituye en 1982 en Gwat (Suiza). En 1986 se funda también en Suiza (Magliaso), una red europea de teólogas que trabajan científicamente, La sociedad europea para la investigación teológica realizada por mujeres (ESWTR). Gracias a la presencia de mujeres españolas significativas en estas organizaciones, junto a inquietudes que se despertaban en muchas, van naciendo también en España en los 80 algunos grupos. Es interesante destacar como nuestra socialización europea es un hecho desde el comienzo.

En 1986 se crea en España el Foro de Estudios sobre la Mujer. Es miembro del Foro Ecuménico de Mujeres Cristianas de Europa. En 1986 nace también 
el primer grupo de Mujeres y Teología. Actualmente, es un movimiento ampliamente extendido, con grupos autónomos y federados por toda España y conocido fuera de sus fronteras. La Asociación de Teólogas Españolas (ATE) que nace en 1992 entra en relación con la iniciativa Sínodo Europeo en 1994 y participó activamente en la realización del Primer Sínodo Europeo de mujeres.

El movimiento Sínodo Europeo nace en los años noventa como un movimiento renovador que pretende dar cabida a un mayor abanico de mujeres, teniendo en cuenta las nuevas realidades de las pertenencias eclesiales. Es ya fruto de la reflexión teológica expresamente feminista y no sólo de la hecha por mujeres:

- Hay muchas mujeres de inspiración cristiana que no se sienten representadas por las iglesias, ni por sus instituciones, aunque sean femeninas. Creen que se empeñan más en defender sus respectivas instituciones y programas que en comprometerse con los asuntos feministas específicos.

- Asimismo, la realidad europea es mucho más plural y compleja. Movimientos migratorios importantes hacen presentes otras realidades religiosas.

- El crecimiento de la llamada indiferencia religiosa da lugar también a una reivindicación de espiritualidades no vinculadas a las religiones tradicionales.

Aunque propiamente la espiritualidad feminista característica de este movimiento se llama espiritualidad política. Desde el comienzo se ha comprometido con la íntima e intrínseca relación existente entre el crecimiento y la transformación personal y la política y justicia social. Que lo personal es político (lema feminista conocido) no sólo significa que los problemas que las mujeres han experimentado como personales y privados son de hecho causados y sólo pueden ser rectificados con una reforma estructural, sino que la transformación social sólo es posible por medio y en base a una transformación personal. Por ello, los apartados en los que los que el movimiento Sínodo trabaja son la economía, la política, el desarrollo personal y la espiritualidad. La espiritualidad feminista comienza con un compromiso que afronta simultáneamente hacia dentro y hacia fuera.

Lo que permite a los demás y a una misma reconocer una espiritualidad sapiencial política son sus frutos:

- conciencia permanente del propio valor, potencial y singularidad;

- sentido sereno pero claro de la propia autoridad; 
- postura intensamente apasionada a favor de la justicia social

- aptitud para estar auténtica e inequívocamente presente

- capacidad para respetar a los demás

- pasión por transformar las relaciones de dominación y

- compromiso por trabajar para reestructurar la colectividad ${ }^{5}$

\section{Primer Sínodo Europeo: Gmunden (Austria 1996)}

Más de mil mujeres, procedentes de 31 países europeos y 14 no europeos, celebraron el Primer Sínodo Europeo de mujeres. Con el lema «Las mujeres por el cambio en el siglo $X X I^{6}$, se quería dejar patente que las mujeres no somos ajenas a todos los cambios y circunstancias que están teniendo lugar en nuestro continente, sino que somos sujetos participativos en todas las áreas. Nuevamente, es preciso hacer visible lo «aparentemente obvio o invisible». Mujeres relevantes y de a pie del mundo de la política, la economía, la teología, los movimientos sociales, organizaciones no gubernamentales, la cultura, las artes trabajaron juntas y compartieron durante los días 21 al 28 de julio de 1996 en ese entorno alpino magnífico que es la ciudad de Gmunden (Austria).

\section{Intereses y acuerdos trabajados}

Según la Declaración final, pueden rastrearse algunos intereses y líneas de fuerza que fueron centrales en los trabajos de este evento.

Hay, por un lado, una incidencia importante en que los derechos de las mujeres son derechos humanos fundamentales, se asume así la Plataforma para la acción de Pekín. Y, por otro lado, existe una condena importante a la guerra, la producción de armamento, la violencia (especialmente violaciones de guerra, la defensa de las minorías), fanatismo, el racismo, demanda de cuidados terapéuticos para las víctimas de estos conflictos. Se reclama asimismo, apoyo a las iniciativas no violentas de las mujeres.

Estamos en un marco en que se quiere escuchar especialmente a los países de Europa del Este y recién se está saliendo de la guerra de la antigua Yugoslavia y firmando algunos acuerdos. Es un aspecto muy determinante, puesto que había grupos de mujeres presentes muy implicadas. Además de estos temas centrales se hacen demandas más concretas en los distintos ámbitos.

5. Diana L. NeU, «Una voz de sabiduría-sofía: terapia/dirección espiritual feminista», Concilium 288(2000), 691-704

6. P. De Miguel (ed.), Europa con ojos de mujer. Primer Sínodo europeo de mujeres, EVD, Estella (Navarra), 1996. 
a. Ámbito político. Se hace especial hincapié en la necesidad de nuevos procedimientos de toma de decisiones en los estados y las instituciones. Y se urge la adopción y el cumplimiento de los instrumentos internacionales sobre Derechos Humanos para poner fin a todas las formas de discriminación. Así como la separación del estado y la religión.

b. En el ámbito económico es central la idea de que las mujeres están en el centro de la economía. En realidad, hacen las dos terceras partes del trabajo. Se pide que se reconozca todo ese el trabajo, tanto pagado como no pagado, en las estimaciones subsidiarias y en las estadísticas oficiales. Se insiste en la resistencia al neoliberalismo y a sus efectos negativos, notorios, por ejemplo, en los recortes de las prestaciones sociales, en los programas de reajuste estructural, en el tráfico de mujeres, el turismo sexual, la emigración y la explotación de la tierra. Se exige en consecuencia modelos alternativos con fórmulas creativas para la organización del trabajo y el tiempo libre, así como la igualdad en el reparto del trabajo. Son decisivos los pequeños pasos que damos en la vida cotidiana, como el poder que ejercemos en nuestras opciones como consumidoras. Se condena expresamente la energía nuclear, aleccionadas por los efectos de Chernobil, y se insta a atender adecuadamente a las víctimas del desastre.

c. Espiritualidad. Se parte el reconocimiento de que las mujeres poseen autoridad espiritual y religiosa en todas las esferas de la vida, así como la rica y creativa labor teológica, educativa, pastoral y litúrgica realizada por ellas Por ello, se exige acceder a todos los ministerios de la iglesia, incluida la ordenación ministerial en la Iglesia Católica. Se define la espiritualidad de las experiencias de las mujeres como una mística y un profetismo nuevos surgidos en la solidaridad y en la lucha por la justicia, la paz y la integridad de la creación. Y se expresa especial respeto por la imaginación creadora de las mujeres que desarrollan estilos de vida y modelos de supervivencia alternativos, por ejemplo entre las mujeres indígenas o las que en Europa y en otras partes del mundo se hallan sumidas en la pobreza.

d. Desarrollo personal. Se da prioridad a la afirmación del derecho de toda mujer a ser dueña de su propio cuerpo y de elegir distintos estilos de vida. Asimismo se insta al reconocimiento del trabajo que realizan las mujeres en las organizaciones no gubernamentales en pro de la justicia, el desarrollo y la paz y la labor expresa de presión para que sean ordenadas las mujeres en todas las iglesias, de modo que 
alcancen finalmente un estatuto igual al de los varones en todos los ministerios. Denunciando así la exclusión sistemática de los puestos de autoridad en la iglesia y en la sociedad de que son víctimas las mujeres. Se urge a las mujeres para que crezca el número de las que intervienen activamente en la política y en el ejercicio de la autoridad religiosa, comprometiéndose así a colaborar en la supresión de estas estructuras injustas.

\section{Segundo Sínodo Europeo: Barcelona 2003}

El Segundo Sínodo europeo de mujeres tuvo lugar en la Universidad Autónoma de Barcelona entre los días 5 al 10 de agosto. Reunió a más de 700 mujeres provenientes de todo el mundo, con el título «Compartir culturas/atreverse con la diversidad» ${ }^{7}$.

Siete ponencias principales y más de cincuenta talleres y seminarios en diferentes lenguas, además de actos culturales, litúrgicos y encuentros de distintas redes formales e informales conformarán la semana de intercambio de este evento. Mujeres de toda condición, de procedencia religiosa y cultural diversa, de más de veinte países del mundo de la teología, la política, las organizaciones sociales, culturales, etc. debatieron y pusieron en común sus experiencias, trabajos, reflexiones y tomaron decisiones de cara a establecer políticas comunes, de acuerdo a sus intereses, para los próximos años.

Muchas mujeres europeas observan los cambios políticos y económicos de Europa y también del mundo con espíritu crítico, quieren influir en estos cambios y participar en los procesos de decisión. Para ello son necesarios encuentros de mujeres de todos los países para intercambiar vivencias y intereses aprendiendo unas de otras a fin de poder elaborar planes de acción conjuntas. Las mujeres desean desarrollar ideas propias y alternativas para mejorar las necesidades vitales y superar las discriminaciones. El sínodo europeo les brinda, para ello, un foro. El término «sínodo» proviene del griego y significa hacer camino con..., un grupo de personas se reúnen para estudiar sus intereses y tomar decisiones al respecto. No es pues lo mismo que un congreso de mujeres. El sínodo europeo de mujeres es un movimiento que quiere completar las discusiones políticas y económicas alcanzando una dimensión espiritual y ética.

Este Sínodo quiere demostrar que una cultura de la diversidad es posible. El hecho de estar reunidas con diferentes puntos de vista y el deseo de

7. P. De Miguel Y Ma Josefa Amell (eds), Atrevir-se a la diversitat, Edit. Mediterrània; Atreverse con la diversidad, EVD, Estella (Navarra), 2004. 
entenderse debe ser el punto central del encuentro. En líneas generales los temas que se tratan están basados en las resoluciones del Primer Sínodo Europeo de mujeres:

- Romper fronteras en Europa.

- Conocer las realidades vitales de las mujeres del Este y del Oeste, del Norte y del Sur.

- Acercarse a las diferentes formas de vida de las mujeres.

- Vivir y compartir parecidas y diversas experiencias religiosas y espirituales.

- Estudiar los derechos de las mujeres y su participación en la toma de decisiones en asuntos de las iglesias, las religiones, la política, la sociedad y los negocios.

El conocimiento de la realidad de Europa del Este fue prioritario en el Primer Sínodo, al elegir Barcelona (España) para el segundo se pretendía una mayor interrelación con las mujeres de Europa del Sur.

\section{Algunos acuerdos y líneas de fuerza trabajadas}

Las europeas estamos reinventando, redefiniendo y renegociando nuestra identidad individual y colectiva como identidad abierta. Esta situación está ensombrecida por el riesgo constante y la incertidumbre. A pesar de que a menudo parece no haber caminos para la negociación en medio del riesgo en que vivimos (desempleo, violencia, desequilibrio ecológico, conflictos bélicos, discriminación, corrupción, miedos, inseguridades...), buscamos alternativas a la situación actual

Padecemos distintos tipos de violencia (sobrecarga de identidad: género, etnia, orientación sexual, religión...; económica, social, política, académica, sexual...) generadora de sufrimiento y rabia. Puesto que no queremos sentirnos víctimas nunca más, decidimos transformar nuestros miedos e iras en pensamiento y acciones creativas.

a. El ámbito político ha de entenderse como el arte del consenso construido mediante el diálogo en la diversidad de intereses. Este tiempo de caos y riesgo nos exige reinventar tantas políticas como respuestas nuevas necesitemos, como garantizar la participación y el liderazgo político de las mujeres o reiterar la denuncia de toda discriminación jurídica, educativa, religiosa (especialmente el tráfico de mujeres y los malos tratos). Y construir sociedades abiertas a la diversidad cultural, sexual, racial y religiosa. 
b. En el ámbito socioeconómico esto se refleja en como el sistema neoliberal lo invade absolutamente todo hasta destruir los fundamentos de nuestra existencia: dañan la tierra, incrementan números de empobrecidos y excluidos y las mujeres somos las más afectadas por este proceso. Por ello se ha de apostar por una mayor calidad de vida, libertad y seguridad económica para todas las mujeres, que haga viable una distribución equitativa de la riqueza y en equilibrio con los recursos naturales. Promover políticas públicas que concilien la vida personal y familiar de las mujeres con el trabajo. Exigir políticas migratorias que proporcionen a las mujeres mejores condiciones de vida y mayor participación democrática.

c. La espiritualidad es importante en nuestras vidas, una espiritualidad diversa en su origen y expresión y conectada con nuestra forma de experimentar la vida como mujeres. Por ello queremos explorar y compartir en red intereses y necesidades espirituales, así como apoyarnos mutuamente en el desarrollo de la autoconciencia y la reflexión y animar a las mujeres de las religiones tradicionales a utilizar aquello que les sea útil y abandonar cuanto les resulte opresivo. Pensar por nosotras mismas con conciencia crítica y desafiar cualquier tendencia de fundamentalismo religioso y denunciar todas las formas de abuso y, particularmente, los abusos sexuales en las comunidades de fe, y a pedir apoyo y compensación para las afectadas. Así como exigir a las religiones/iglesias: formas alternativas de culto, la utilización de un lenguaje inclusivo, también para la divinidad, que acepten y acojan en vez de culpar y condenar, que permitan acceder a las mujeres a todos los ministerios

d. En el ámbito del desarrollo personal se es consciente de que para descubrir identidades individuales y crecer como mujeres se han de fortalecer nuestra autoestima, confiar en nuestras experiencias, y tomar nuestras propias decisiones por medio del desarrollo de las redes de mujeres que nos ayudan en nuestra vida personal, relacional, laboral, valorando especialmente la solidaridad; apoyadas en aquellas que nos precedieron transmitirlas a las nuevas generaciones. Y exigir a las instituciones políticas y educativas, a las iglesias y a los medios de comunicación, un cambio de estructuras que visibilicen a las mujeres y las integren en los procesos de control y toma de decisiones. 


\section{Conclusión: no dejar a la diosa Metis en el vientre de Zeus}

Metis es la personificación de los distintos modos aplicados del conocimiento y la acción. Metis connota la habilidad de comprender una situación de forma intelectual y actuar con sabiduría y pericia. Metis fue una diosa preolímpica de la sabiduría, quien tras ser acosada por Zeus se convirtió en su primera esposa. Fue ella quien proporcionó a Zeus los medios gracias a los cuales el dios pudo llegar a gobernar todas las divinidades del monte Olimpo.

Era hija de titanes: Tetis, la diosa de la luna, y Océano, cuyo reino era una extensa masa de agua que rodeaba la tierra. Como titán, formaba parte de esa vieja orden dominante de divinidades que Zeus intentaba derrocar. Al final, la atrapó. Cuando Metis estaba embarazada de Zeus, un oráculo de la tierra le dijo al padre que ese hijo sería una niña, y que si concebía de nuevo, Metis daría a luz un hijo cariñoso que lo suplantaría. Zeus se acercó a Metis con astucia y zalamería para seducirla y distraerla. Consiguió llevarla al lecho, donde la engañó para que se hiciera pequeña y se la tragó. Aunque Zeus afirmaba que le aconsejaba desde su vientre. Por consiguiente, el dios la incorporó a su persona y tomó sus atributos y su poder como propios, incluyendo el parto. Zeus alumbró a Atenea por la cabeza ${ }^{8}$.

Atenea es la hija del padre y cómoda con el Patriarcado, pero cuando advierte que la dedicación enorme que ha dedicado a mantenerlo no es correspondida con sus expectativas, experimenta una profunda traición y desengaño que podría significar una iniciación a la sabiduría que haría revivir a Metis.

Esta metáfora tan sugerente de Metis engullida por Zeus nos da para repensar creativamente tantas realizaciones de mujeres, individual y colectivamente tragadas por el patriarcado al apropiarse éste de sus recursos, ideas y atributos. Pero la Sabiduría (Metis) emerge una y cien veces en la historia con distintas formas para recordarnos que podemos avivarla siempre que desenterremos nuestros relatos y acciones.

En estas páginas quiero insistir en la necesidad de escuchar las historias recientes y coetáneas de muchas mujeres, cuyas vidas cotidianas son vivos ejemplos de resistencia. Tenemos que generar o recuperar estos testimonios, porque así no caeremos en la tentación de pensar que sus -nuestros- logros cotidianos han sido y son irrelevantes ${ }^{9}$.

Emergió con la forma histórica de acción de cuáqueras sufragistas: «Gritaban a voz en cuello, las metían en la cárcel, eran despreciadas por las mujeres

8. J.Shinoda BOLEN, Las diosas de la mujer madura. Arquetipos femeninos a partir de los cincuenta, Kairós, Barcelona, 2003.

9. Anabella BARROSO, «Mujeres, resistencia y vida cotidiana» en Pilar de Miguel (ed), Espiritualidad y fortaleza femenina, DDB, Bilbao, 2006. 
«honradas», denunciadas por los clérigos, obligadas a comer por la policía... No eran «simpáticas». Eran vuestras abuelas. Eran las sufragistas. Y tenían razón» (Joan Chittister).

Emerge también en cada relato que dota a las mujeres de poder de decisión, que reconoce sus atributos y recursos, y los pone a funcionar autónomamente para su bien y por tanto para el bien de la humanidad. El libro bíblico de Judit, de tradición judeocristiana, es paradigmático en mi opinión en este sentido

\section{Judit}

Judit está viuda y no tiene hijos. Desde la perspectiva dominante, esto es una gran deshonra en una mujer judía de la época. Una mujer sin marido y sin hijos quedaba excluida de la escena de los existentes. No se era ni se existía como persona-mujer, salvo en esos roles. Pero, se había ceñido de sayal y se vestía vestidos de viuda, ayunaba, vivía en su casa; es decir, cumplía perfectamente con las expectativas reinantes sobre una viuda.

Vive en Betulia (nombre simbólico que significa la joven o la muchacha) y Holofernes ha puesto cerco a esta ciudad desde hace 34 días. El pueblo está muerto de sed y sus jefes le conceden cinco días a Dios para que responda con la lluvia. De fondo está la teología de la retribución; el pueblo piensa que ha obrado mal y que Dios le castigará y sus jefes esperan milagros. No es difícil establecer una comparación entre la situación de Betulia y la de Judit. Ella tampoco tiene muchas posibilidades de vida plena, de futuro. A ambas les falta la fuente de la vida, tienen sed.

Judit se indigna al enterarse de que los jefes tienen tan poca «memoria». No está en absoluto de acuerdo con el «análisis de la realidad» que hacen. Según su propia experiencia, su Dios no es el dios de la lluvia. La experiencia de Dios que Judit tiene es otra, tiene que ver con la gratuidad y la confianza, es la que dice «esperemos más bien confiados en que quiera salvarnos, y escuchará nuestra súplica si quiere hacerlo». Dicho esto, el trabajo es asunto nuestro.

¿Quiénes son ellos para poner a Dios a prueba, ellos que ni siquiera conocen lo profundo del corazón humano? -y las ansias de liberación de las viudas, de los «sin futuro», parece querer continuar...- Es el Dios que los sacó de Egipto, es el Dios de la liberación, experiencia fundante de la fe hebrea. Deberían estimular al pueblo a hacerse responsables activamente frente a una situación de peligro en lugar de resignarse teológicamente en una figura divina falsa.

Lleva «cercada» por el enemigo mucho tiempo: 3 años y 4 meses, es decir, 40 meses asumiendo el papel que irremediablemente se le había asignado. 
El mismo tiempo que Betulia, 34 días más los 5 que le dan sus jefes a Dios de plazo. Este 40 le suena a Judit a los años de desierto y a la liberación que precedió a su pueblo. Moisés hizo algo. Ella hace memoria y se da cuenta que puede hacer algo por su emancipación y la de su pueblo. Al día siguiente, el 40 , era tiempo de despertar a una nueva vida.

Lo que se espera que las mujeres hagan por su pueblo es traer hijos al mundo para hacer al pueblo poderoso y nutrir los ejércitos de guerreros. Parece que el sistema falla. Ni ella tiene hijos y además está viuda y el pueblo no parece dispuesto a guerrear para defenderse. A lo mejor hay otra manera. Judit recuerda que para su Dios «el poder no está en el número, ni su señorío se apoya en los guerreros sino que es el Dios de los humillados, ayuda de los pequeños, defensor de los débiles, protector de los abandonados». En su oración, Judit evoca al Dios de su pueblo, pero también revive cual es el deseo de Dios frente a lo sucedido con sus antepasadas humilladas. Hace memoria específica de género. Ese Dios es también quien vengó a Dina y a todas las mujeres humilladas y esclavizadas:

Señor, Dios de mi padre Simeón, al que pusiste una espada en la mano para vengarse de los extranjeros que desfloraron vergonzosamente a una doncella, la desnudaron para violentarla y profanaron su seno deshonrándola. Aunque tú habías dicho: «No hagáis eso», lo hicieron...

Sea una mujer, por esta vez, no la humillada sino la liberadora: Que mi mano de mujer quebrante su arrogancia. Después de asumir su ser de mujer y su situación de «cercada-sitiada» y de solidarizarse con los otros «cercados» o sitiados, utilizando los instrumentos a su alcance -los que le permite el momento, su belleza, inteligencia, mi lengua seductora- consigue salvar la situación.

Todos y todas celebran su victoria final, pero ella no pierde la oportunidad de explicar que no es sólo lo aparente, lo que ven, que Holofernes ya no tiene poder; sino que ella ha afrontado también creativamente su situación y se autonombra madre del pueblo, ella ha ejercido la maternidad de otra manera ${ }^{10}$. Puede decirse que ha revivido a Metis...y así....

10. El libro de Judit es un ejemplo paradigmático pero especialmente se cita el capítulo 9. Puede verse un estudio más amplio en www.efeta.org/galeria 


\section{Referencias bibliográficas}

LAGARDE, M. «Aculturación feminista» en:

www.geocities.com/litertulia/acultur_mlagarde.htm. 20/04/01.

De Miguel, P. (ed.) Europa con ojos de mujer. Primer Sínodo europeo de mujeres, Estella (Navarra): EVD, 1996.

- y Amell, M ${ }^{\mathrm{a}} \mathrm{J}$. (eds.), Atrevir-se a la diversitat, Barcelona: Mediterrània; castellano: Atreverse con la diversidad, Estella (Navarra): EVD, 2004.

-, (ed.). Espiritualidad y fortaleza femenina, Bilbao: DDB, 2006

_, (ed.). En qué creen las mujeres. Creyendo y creando, Bilbao, DDB, 2007.

NEU, D. «Una voz de sabiduría-sofía: terapia/dirección espiritual feminista», Concilium 288 (2000), pp. 691-704.

SHINODA BOLEN, J. Las diosas de la mujer madura. Arquetipos femeninos a partir de los cincuenta, Barcelona: Kairós, 2003. 